\title{
Vaccination with Different M2e Epitope Densities Confers Partial Protection against H5N1 Influenza A Virus Challenge in Chickens
}

\author{
Xintao Zhang Ming Liu Chunguo Liu Jinling Du Weilin Shi Encheng Sun \\ Hongtao Li Jianhui Li Yun Zhang \\ Animal Influenza Laboratory of the Ministry of Agriculture, and State Key Laboratory of Veterinary Biotechnology, \\ Harbin Veterinary Research Institute, Chinese Academy of Agricultural Sciences, Harbin, PR China
}

\section{Key Words}

Avian influenza virus $\cdot \mathrm{H} 5 \mathrm{~N} 1 \cdot$ M2e gene $\cdot$

Universal vaccines

\begin{abstract}
Objective: Currently, research is focused on universal influenza vaccines based on various ectodomains of the influenza matrix protein 2 (M2e). Such vaccines are tested mostly using mouse-adapted influenza viruses and in mouse or ferret models. The aim of this study was to investigate in a chicken model the protective efficacy of vaccines based on aviantype M2e at different epitope densities. Methods: On the basis of the optimized avian-type M2e gene, recombinant plasmids that contained tandem copies of different M2e were constructed and expressed in Escherichia coli. The expression and immunogenicity of the proteins were confirmed by SDS-PAGE and Western blot, as well as immunofluorescence assay and enzyme-linked immunosorbent assay. Animals were immunized with fusion proteins emulsified with an appropriate adjuvant and then infected with highly pathogenic influenza virus of A/chicken/Guangdong/04 (H5N1). Antibody levels, survival rate and weight loss were investigated. Results: Multiple copies of M2e were highly expressed; higher epitope density engendered better protection but there was not a linear increase. Among the
\end{abstract}

fusion proteins, the MBP-3.M2e fusion protein showed the best protective efficacy. Conclusions: This study has provided evidence that the immune response to avian-type M2ebased subunit vaccines was greater in chickens than that in mice. In addition, higher M2e epitope density can yield better protection, but not in a linear fashion.

Copyright $\odot 2011$ S. Karger AG, Basel

\section{Introduction}

Avian influenza (AI), although a vaccine-preventable disease, remains a significant cause of substantial morbidity and mortality in domestic poultry in China. Vaccination with various inactivated and live virus-vectored vaccines is the major tool used to control $\mathrm{AI}$, together with culling of infected poultry [1]. Currently, nearly all licensed influenza vaccines are based on hemagglutinin (HA) as the dominant antigen; however, their shortcomings are revealed to some degree by the requirement for long-term application of these vaccines, together with the influence of environmental factors. For example, the possible occurrence of antigenic drift and even antigenic shift in the dominant viral antigens of the HA leads to a mismatch between the vaccines and field viruses in the presence of endless selection pressure [2]. Moreover, the

\section{KARGER}

Fax +4161306 1234

E-Mail karger@karger.ch

www.karger.com
(C) 2011 S. Karger AG, Basel

0300-5526/11/0545-0290\$38.00/0

Accessible online at:

www.karger.com/int
Ming Liu

Animal Influenza Laboratory of the Ministry of Agriculture

Harbin Veterinary Research Institute, CAAS, 427 Maduan Street

Harbin 150001 (PR China)

Tel. +86 1894606 6068, Fax +86 4518273 3132, E-Mail liuming04@126.com 
cross-protective ability of vaccines that rely on HA may be unreliable once new strains overcome the species barrier. Therefore, the available licensed vaccines cannot guarantee that a new influenza pandemic could be controlled effectively. Additional anti-infection strategies and novel approaches are urgently required to resolve the above dilemma.

Research on universal influenza vaccines that are based on the conserved internal proteins of influenza virus, such as nucleoprotein and matrix, is followed with interest [3]. Vaccines based on the ectodomain of influenza matrix protein 2 (M2e) have attracted extensive attention and have gradually become a focal point of research. Recently, various specific vaccine constructs containing M2e have been explored and tested for immunogenicity and protection. Given the small size of native M2e and its almost complete lack of immunogenicity, M2e has been attached to a variety of carriers [47], such as the hepatitis B virus ( $\mathrm{HBc}$ ) or applied with innovative adjuvants [8-10]; both strategies provoke a stronger immune response. Some lines of vaccines have been shown to protect various animal models against certain strains of influenza, mostly by eliciting heterosubtypic immunity to varying degrees $[11,12]$. Therefore, M2e-based vaccines are considered to be promising candidates to provide a strategy for or to supplement the control of unexpected outbreaks or pandemics. However, most M2e-based vaccines have been developed for application as human influenza vaccines, and have been tested largely in mouse or ferret models; evaluation in avian models has been limited.

M2e, which is only 23 amino acids in length, is highly conserved and undergoes noticeably little sequence variation among AI viruses (AIV) [13, 14]. Comparisons among M2e sequences of human and AI A viruses show that the nine $\mathrm{N}$-terminal amino acids of the avian-type M2e consensus sequence are identical to those of the human-type M2e consensus sequence [15], but the latter being up to 5 positions different from the former [16]. Furthermore, an experiment has demonstrated that these sequences showed incomplete reciprocal cross-reaction [17], which implies a potential discrepancy in their immunogenicity and protective efficacy. An early study has shown a close correlation between epitope density and epitope-specific humoral immune responses in a mouse model [18], but it is not clear whether a high epitope density of avian-type M2e could function in a chicken model, too. At the same time, it is worthwhile to compare the level of humoral immunity raised in mice and chickens.

M2e-Based Vaccines against H5N1
This study was designed to determine the protective efficacy of M2e-based subunit vaccines including different numbers of M2e tandem copies. The n•M2e subunit vaccines were established and tested by measuring their ability to induce an antibody response, protective efficacy and viral shedding in vaccinated chickens following virus challenge. Chickens were challenged with $\mathrm{H} 5 \mathrm{~N} 1$ influenza virus to assess protective efficacy, and an additional parallel experiment in mice extended these findings.

\section{Materials and Methods}

Viruses and Cells

CG [AIV A/chicken/Guangdong/04 (H5N1)] was propagated in 10-day-old eggs. Madin-Darby canine kidney cells (MDCK) were maintained in MEM containing 5\% fetal calf serum at $37^{\circ}$ in $5 \% \mathrm{CO}_{2}$. All operations with $\mathrm{H} 5 \mathrm{~N} 1$ virus were performed in biosafety level 3 facilities.

Peptide and Chemical Reagents

The M2e peptide, N-SLLTEVETPTRNEWECRCNDSSD (amino acids 2-24), was synthesized at Biotech Bioscience \& Technology (Shanghai, China). Freund's adjuvant was obtained from Sigma-Aldrich (St. Louis, Mo., USA) and Montanide ${ }^{\mathrm{TM}}$ ISA 70 M VG adjuvant was obtained from Seppic (Paris, France). The pGEM-T easy vector plasmid and pMAL-c2x plasmid were obtained from Promega (Madison, Wisc., USA) and New England Biolabs (Ipswich, Mass., USA), respectively. The monoclonal antibody 14C2 specific for M2 protein was purchased from Santa Cruz Biotechnology (Santa Cruz, Calif., USA).

Preparation of Fusion Proteins with Various Epitope Densities

The E. coli maltose binding protein (MBP) fusion proteins bearing various epitope densities were constructed by the epitope cumulation recombinant technique described previously [19]. In brief, two oligonucleotide chains (M2e-F1 and M2e-R1) and primers (M2e-F2 and M2e-R2) were synthesized to construct the $\mathrm{M} 2 \mathrm{e}$ gene by overlapping polymerase chain reaction (PCR; table 1). The PCR products were cloned directly into the pGEM-T easy vector (Promega), and the positive clone was named pGEMM2e. For simpler manipulation of the digested fragments in the following process, the 294-bp BglII and XhoI double-digested M2 gene fragment, amplified with gene-specific primers M2-F1 and M2-R1, was inserted in the above-constructed pGEM-M2e plasmid by the same digestion treatment, and the screened positive recombinant plasmid was named pGEM-T1. The BglII and XhoI double-digested pGEM-T0 and the lower molecular weight (MW) band of the BamHI and XhoI double digests of pGEM-M2e were purified and ligated with T4 DNA ligase (New England Biolabs, Ipswich, Mass., USA). The screened positive clone was named pGEM-T2, and contained two and one copies of M2e. Subsequently, pGEM-T3, pGEM-T6, pGEM-T9 and pGEM-T12 were constructed by repeating the above process, respectively (fig. 1). For all five recombinant plasmids, the lower MW band of the $B a m \mathrm{HI}$ and BglII double digests was subcloned into the BamHI

Intervirology 2011;54:290-299 
Table 1. Templates and primers used in overlapping PCR

\begin{tabular}{ll}
\hline M2e-F1 & 5' -gcgcgggatcctctctgctgaccgaagttgaaaccccgacccgtaacgaatgggaagg-3' \\
M2e-R1 & 5' -gttatcaagatctagaaccaccaccaccgtcagaagagtcagagcaacggccttcccattcgttacgggt-3' \\
M2e-F2 & $5^{\prime}$-gtgcgcggatcc-3' \\
M2e-R2 & $5^{\prime}$-ctcgagttatcaagatct-3' \\
M2-F1 & $5^{\prime}$-ggcagatctagtcttctaaccgaggtcgaaacgcctaccagaagcgaatggga-3' \\
M2-R1 & $5^{\prime}$-aatctcgagttactccaattctatgttgaca-3' \\
M2e & N-M S L L T E V E T P T R N E W E G R G S D S S D-C
\end{tabular}

$\mathrm{F}=$ Forward primer; $\mathrm{R}=$ reverse primer. The underlined nucleotides represent the restriction endonuclease recognized sites. M2e is the amino acid sequence of the extracellular domain of matrix protein 2 of H5N1 influenza A virus.

cleaved prokaryotic expression plasmid pMAL-c2x. The recombinant plasmids with the desired orientation were sequenced to confirm their identity. Component cells of E. coli strain BL21 were then transformed with the recombinant plasmids, and target protein expression was induced by $0.3 \mathrm{~mm}$ isopropylthiogalactoside in Luria-Bertani medium with ampicillin $(50 \mu \mathrm{g} / \mathrm{ml})$ for $12 \mathrm{~h}$ at $16^{\circ}$. The control MBP protein and all other fusion proteins bearing variable $\mathrm{M} 2 \mathrm{e}$ epitope density were purified using the $\mathrm{pMAL}$ protein fusion and purification system (New England Biolabs). They were then quantified using an ultraviolet spectrophotometer (Ultrospec3000; Pharmacia Biotech, Piscataway, N.J., USA).

\section{SDS-PAGE and Western Blotting Assays}

Protein extracts from BL21 cells were mixed with an equal volume of sample buffer, boiled for $10 \mathrm{~min}$ and separated by SDSPAGE. The gels were stained by standard methods using Coomassie brilliant blue to visualize the protein bands. At the same time, nitrocellulose membranes were incubated with blocking buffer (Rockland, Gilbertsville, Pa., USA) overnight at $4^{\circ}$, and the membranes were probed with anti-MBP monoclonal antibody (1:5,000 dilution; New England Biolabs) and M2 monoclonal antibody 14C2 (1:5,000 dilution; Santa Cruz Biotechnology), respectively. Finally, the nitrocellulose membranes were reacted with HRP-labeled goat anti-mouse IgG conjugate (1:5,000 dilution; Rockland) for $2 \mathrm{~h}$ at $37^{\circ}$, and the fusion proteins were visualized with the Odyssey infrared imaging system (LI-COR Biosciences, Lincoln, Nebr., USA).

\section{Animals, Immunization and Challenge}

A prime-boosting regimen was applied to all immunizations. Nine-week-old female BALB/c mice $(\mathrm{n}=42)$ were randomized to seven groups of 6 mice per group. Six groups were immunized intraperitoneally with $50 \mu \mathrm{g}$ of antigen per mouse; the seventh group, which served as the negative control, was injected with adjuvant in $1 \times$ PBS only. Six-week-old specific pathogen-free (SPF) chickens $(n=56)$ were housed in HEPA-filtered isolators and were randomized to seven groups of 8 chickens per group. The chickens underwent the same protocol, but each was administered 150 $\mu \mathrm{g}$ of fusion protein emulsified with Montanide ISA $70 \mathrm{M}$ VG adjuvant (Seppic). In the mouse model, complete Freud's adjuvant (Sigma-Aldrich) was used in the primary immunization, while for the two booster immunizations, incomplete Freud's adjuvant
(Sigma-Aldrich) was chosen. All adjuvants were used according to the manufacturer's directions, and three immunizations were made at 3-week intervals.

Chickens and anesthetized mice were challenged intranasally with $1 \times 10^{6} 50 \%$ egg-infective doses of CG, which was conducted in HEPA-filtered isolators. Survival studies of the animal models were performed and the time to death was recorded. Oropharyngeal and cloacal swabs were collected from the chickens every day after challenge for the evaluation of virus titration, and at the same time mortality and clinical signs were monitored daily for 2 weeks after infection. All procedures involving live animals were approved by the Ministry of Agriculture, PR China.

\section{Serum Collection}

Blood was obtained from euthanized mice by the amputation of the tail, and from chickens by phlebotomy of the wing vein. The precipitate was pelleted in a microcentrifuge after clotting for $1 \mathrm{~h}$ at $37^{\circ}$, and the serum was removed and stored at $-20^{\circ}$ until use.

\section{Indirect Immunofluorescence Assay}

The immunofluorescence assay was performed to evaluate the bioactivity of the expressed M2 protein qualitatively. In brief, confluent MDCK cell monolayers were infected with $100 \mathrm{TCD}_{50}$ of CG and incubated for $12 \mathrm{~h}$ at $37^{\circ}$. The wells were washed three times with PBS containing 0.05\% (V/V) Tween 20 (PBST), fixed in $4 \%(\mathrm{~W} / \mathrm{V})$ paraformaldehyde for $30 \mathrm{~min}$ at room temperature and permeabilized in $0.5 \%(\mathrm{~V} / \mathrm{V})$ Triton $\mathrm{X}-100$ for $20 \mathrm{~min}$ at room temperature. Cells were washed with $1 \times$ PBST, then incubated with murine sera (1:100 dilution, $100 \mu \mathrm{l} /$ well) for $2 \mathrm{~h}$ at $37^{\circ}$. After washing, the plates were incubated with FITC-labeled goat antimouse IgG conjugate (Pierce Biotechnology, Rockford, Ill., USA) for $45 \mathrm{~min}$ at $37^{\circ}$. The plate was washed three times with $1 \times \mathrm{PBST}$ and fluorescence was visualized using a Nikon FXL microscope.

\section{Different Indirect ELISA Procedures}

The M2e-MAP peptide (0.5 $\mu \mathrm{g} /$ well) was coated onto an ELISA plate (Jet Biofil, Guangzhou, PR China), and the plate was incubated overnight at $4^{\circ}$. The plate was washed three times with $1 \times$ PBST then blocked with 5\% (W/V) skimmed milk for $1 \mathrm{~h}$ at $37^{\circ}$. After washing, $100 \mu \mathrm{l} /$ well of the diluted chicken (1:100 dilution) and mouse sera (1:800 dilution) in blocking solution were added and incubated for $2 \mathrm{~h}$ at $37^{\circ}$, respectively. After washing, 


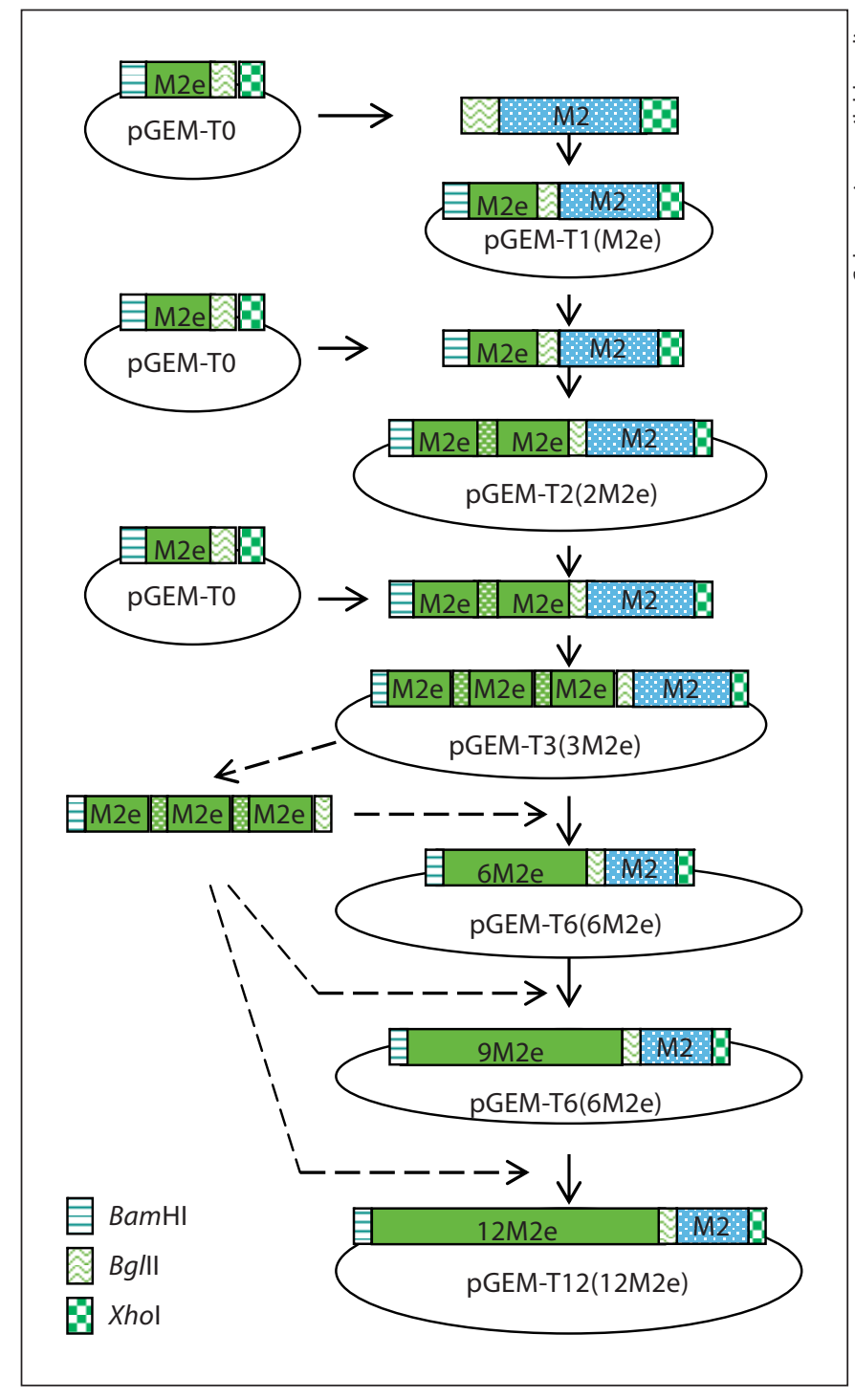

Fig. 1. Schematic diagram of the construction of the M2e tandem copies.

the plates were incubated with $50 \mu \mathrm{l}$ of alkaline phosphatase-conjugated rabbit anti-chicken IgG conjugate (1:10 ${ }^{4}$ dilution), and HRP-conjugated goat anti-mouse IgG conjugate (1:5,000 dilution) was added at $37^{\circ}$ for $1.5 \mathrm{~h}$, respectively. After washing, $p$ nitrophenyl phosphate (Shanghai Ecust Biomedicine, Shanghai, PR China) substrate and $o$-phenylenediamine (Shanghai Ecust Biomedicine) substrate were added for $10 \mathrm{~min}$ at room temperature, respectively. The reactions were stopped with $0.5 \mathrm{M} \mathrm{NaOH}$ and $1 \mathrm{M} \mathrm{H}_{2} \mathrm{SO}_{4}$, respectively. $\mathrm{OD}_{405 \mathrm{~nm}}$ and $\mathrm{OD}_{490 \mathrm{~nm}}$ were read using a microplate spectrophotometer.

In addition to the above indirect ELISA, an MDCK cell ELISA and purified virus particle ELISA were used to test whether sera from chickens and mice could interact with the pathogen proteins. In brief, confluent MDCK cell monolayers were incubated with $100 \mathrm{TCD}_{50}$ of CG in DMEM without FCS for $1 \mathrm{~h}$ at $37^{\circ}$, then $200 \mu \mathrm{l}$ of complete medium was added and incubated overnight at $4^{\circ}$. The subsequent procedures were the same as for the above ELISA.

\section{Statistical Analysis}

Statistical analysis was performed using one-way ANOVA in SPSS 11.5 (SPSS, Chicago, Ill., USA). For all comparisons, differences were considered significant when $\mathrm{p}$ values were $<0.05$.

\section{Results}

The Plasmids Were Constructed Successfully, and the Fusion Proteins Were Expressed Well and Showed Good Immunogenicity

The five plasmid constructions were first identified by BamHI and HindIII digestion (fig. 2), and the sequence determination showed that the constructions were also correct. Efficient expression of fusion proteins was obtained by induction for $12 \mathrm{~h}$ at $16^{\circ}$; the expression of the proteins was evaluated by SDS-PAGE (fig. 3a). The purification of the fusion proteins was achieved with the pMAL protein fusion and purification system (fig. 3b); Western blot demonstrated that the fusion proteins possessed good immunogenicity (fig. 4), and the protein bands were readily visible.

\section{Sera from Chickens and Mice Could Recognize the \\ Native M2 Protein}

To investigate whether sera from chickens and mice recognized the native M2 protein, ELISA was performed using wells coated with CG-infected MDCK cells and purified virus particles. The results indicated that sera from chickens and mice immunized with the five kinds of fusion protein bound especially to virus-infected MDCK cells and virus particles (fig. 5). This demonstrated that anti-M2e serum antibodies recognized native $\mathrm{M} 2$ protein expressed on the surface of CG-infected cells. Furthermore, the immunofluorescence assay showed that the murine sera could react with native M2 proteins expressed on MDCK cells (fig. 6).

Increased M2e Epitope Density Enhances Specific IgG Antibody Titer in Humoral Immunization, but Not in a Linear Fashion

The M2e-specific IgG antibody titers were measured by a different indirect ELISA in which the M2e-MAP peptide, the CG-infected MDCK cells and the purified virus particles were coated as antigen, respectively. Unexpectedly, higher M2e epitope density stimulated a 
Fig. 2. Double digests of the pMAL-n $\cdot M 2 e$ recombinant plasmids by $B a m \mathrm{HI}$ and HindIII in 4\% (a) and $1 \%$ agarose gel (b). Lane $1=$ p-M2e; lane $2=$ p-12.M2e; lane $3=\mathrm{p}-9 \cdot \mathrm{M} 2 \mathrm{e} ;$ lane $4=\mathrm{p}-6 \cdot \mathrm{M} 2 \mathrm{e} ;$ lane $5=$ p-3·M2e; $\mathrm{M}_{1}=20$-bp DNA ladder marker; $\mathrm{M}_{2}=$ DNA marker DL2000.

Fig. 3. Identification of the MBP-n・M2e fusion proteins (a) and purified fusion proteins (b) by SDS-PAGE. a Samples from five fusion proteins induced by isopropylthiogalactoside. Lane $1=$ Non-induced pMAL-c2X; lane 2 = MBP-M2e; lane $3=$ MBP-3 $\cdot$ M2e; lane $4=\mathrm{MBP}-6 \cdot \mathrm{M} 2 \mathrm{e}$; lane $5=\mathrm{MBP}-9 \cdot \mathrm{M} 2 \mathrm{e}$; lane $6=\mathrm{MBP}-12 \cdot \mathrm{M} 2 \mathrm{e}$. b Purified MBP and five fusion proteins. Lane 1 = Purified MBP; lane 2 = purified $\mathrm{MBP} \cdot \mathrm{M} 2 \mathrm{e}$ protein; lane 3 = purified $\mathrm{MBP}$ $3 \cdot \mathrm{M} 2 \mathrm{e}$ protein; lane $4=$ purified $\mathrm{MBP}$ $6 \cdot \mathrm{M} 2 \mathrm{e}$ protein; lane $5=$ purified MBP$9 \cdot \mathrm{M} 2 \mathrm{e}$ protein; lane $6=$ purified MBP$12 \cdot$ M2e protein; $M=$ protein $M W$ marker 0671 (Fermentas, St. Leon-Rot, Germany).
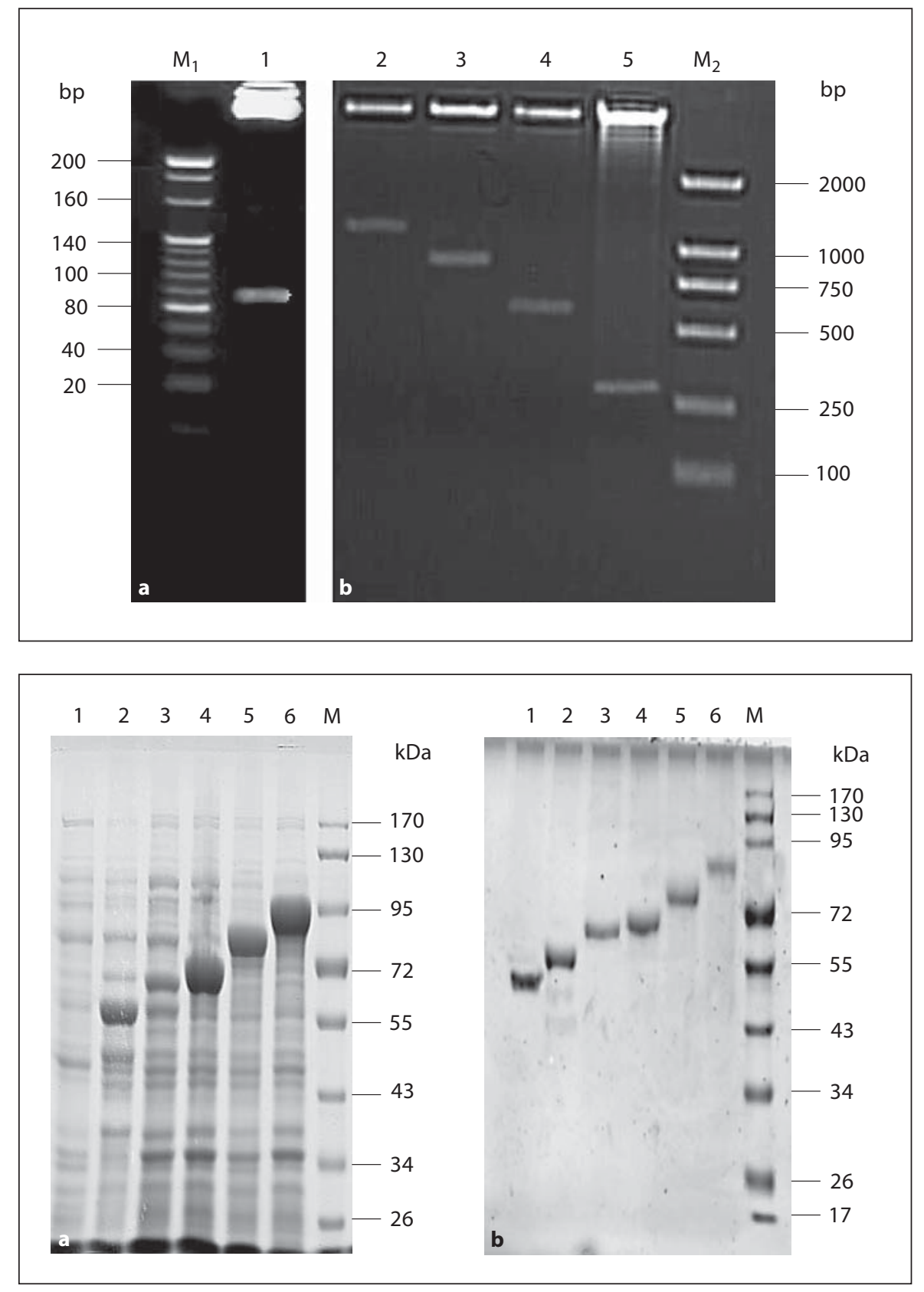

higher level of antibody, but the M2e-specific antibody titers in both chickens and mice were not completely dependent on the degree of M2e epitope density in the fusion proteins, in the case of the prime-boosting protocol. The antibody levels in all groups showed an increase compared with the negative control, and an increase in epitope density enhanced the specific IgG antibody titers, but not in a linear fashion. The levels of specific IgG antibody in the various groups were not significantly dif- ferent in both the chicken and mouse models $(\mathrm{p}>0.1)$. However, the MBP-3·M2e group showed higher antibody titers than the other groups in both models (fig. 5). In the chicken model, the specific IgG antibody levels were higher than, but not significantly different from those in the mouse model ( $p>0.1$; fig. 5a, b). In addition, the antibody level in the MBP-3·M2e group was significantly different from that of the negative control in the chicken model $(\mathrm{p}<0.05)$. 
Fig. 4. Identification of the MBP-n·M2e purified fusion proteins by Western blot. The fusion proteins were checked by MBP monoclonal antibody (a) and M2-specific monoclonal antibody 14C2 (b). Lane $1=$ MBP protein; lanes $2-6=$ the purified MBP-n $\cdot M 2 e$ fusion proteins $(\mathrm{MBP} \cdot \mathrm{M} 2 \mathrm{e}$, MBP-3·M2e, MBP-6•M2e, MBP-9·M2e, $\mathrm{MBP}-12 \cdot \mathrm{M} 2 \mathrm{e}) ; \mathrm{M}=$ protein $\mathrm{MW}$ marker 0671 (Fermentas).

Fig. 5. Antibody levels in sera from SPF chickens (a-c) and BALB/c mice (d-f). ELISA plates were coated with purified influenza virus particles (a, d), M2e-MAP peptide (b, e) and the expressed M2 protein on MDCK cells (c, f). Asterisks indicate significantly increased antibody levels compared with the negative control $(\mathrm{p}<$ 0.05).

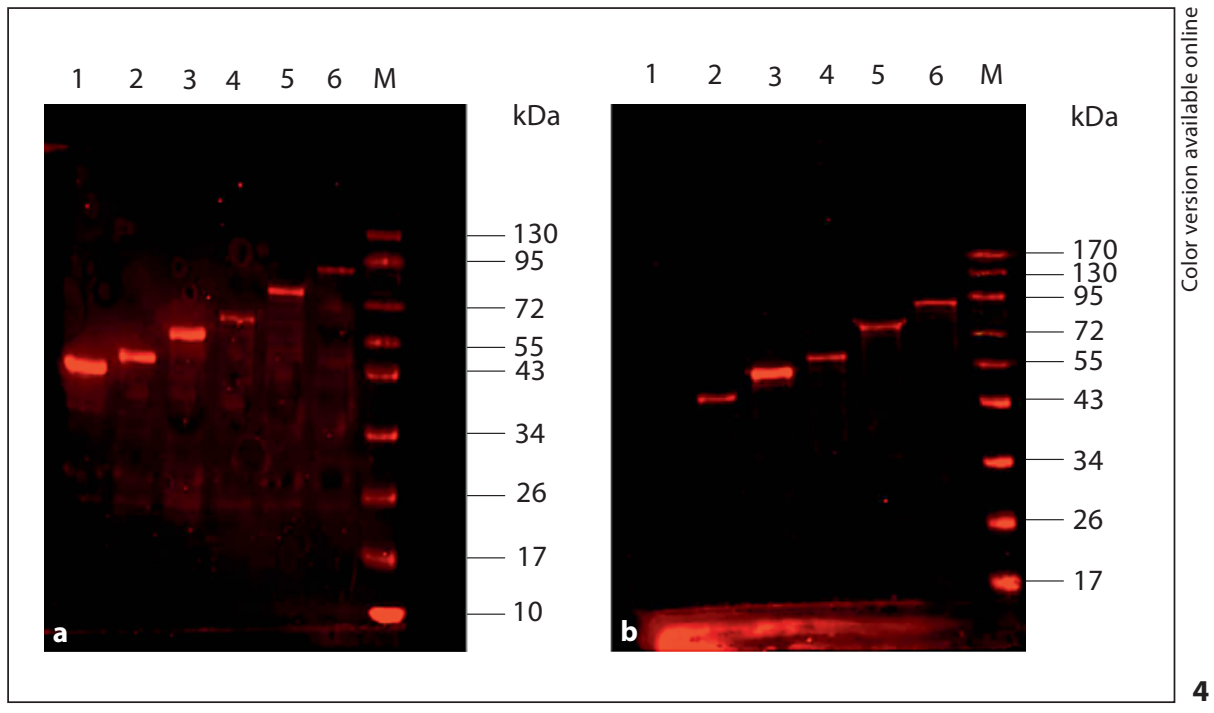

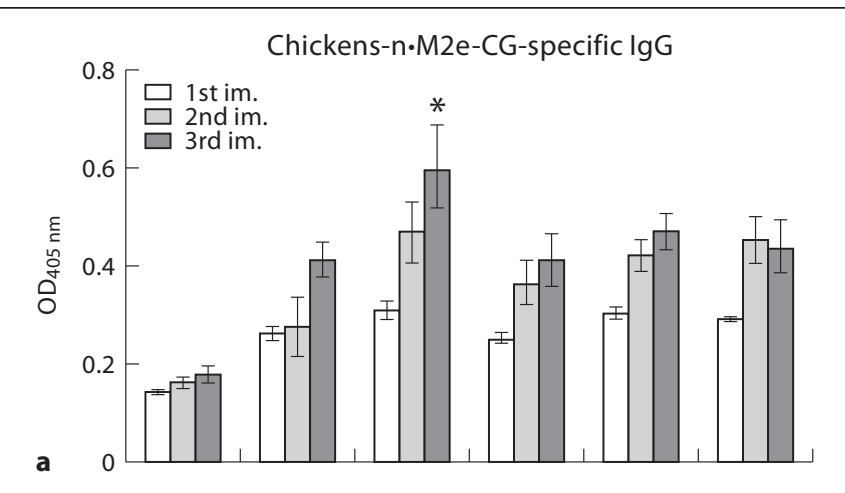
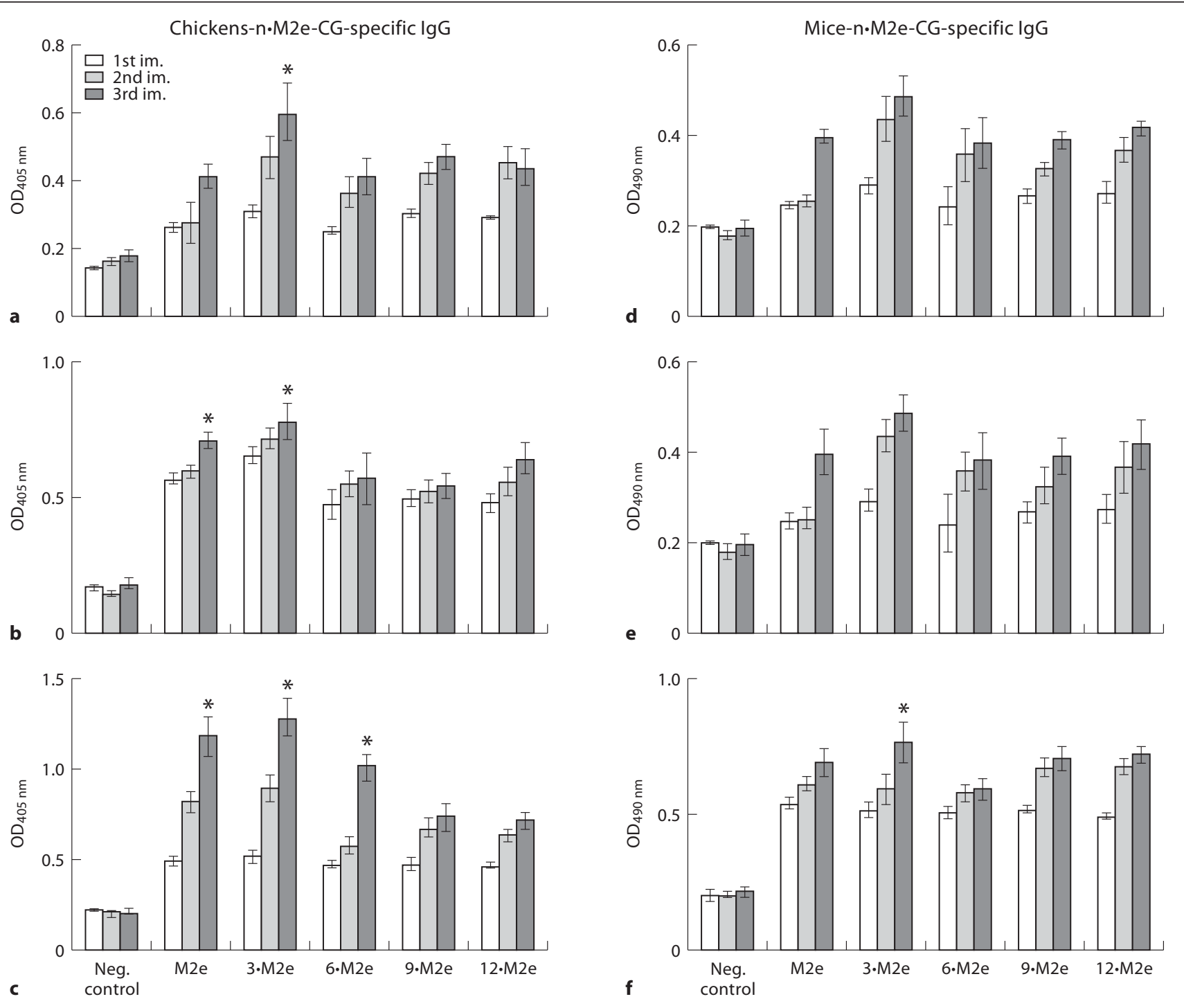

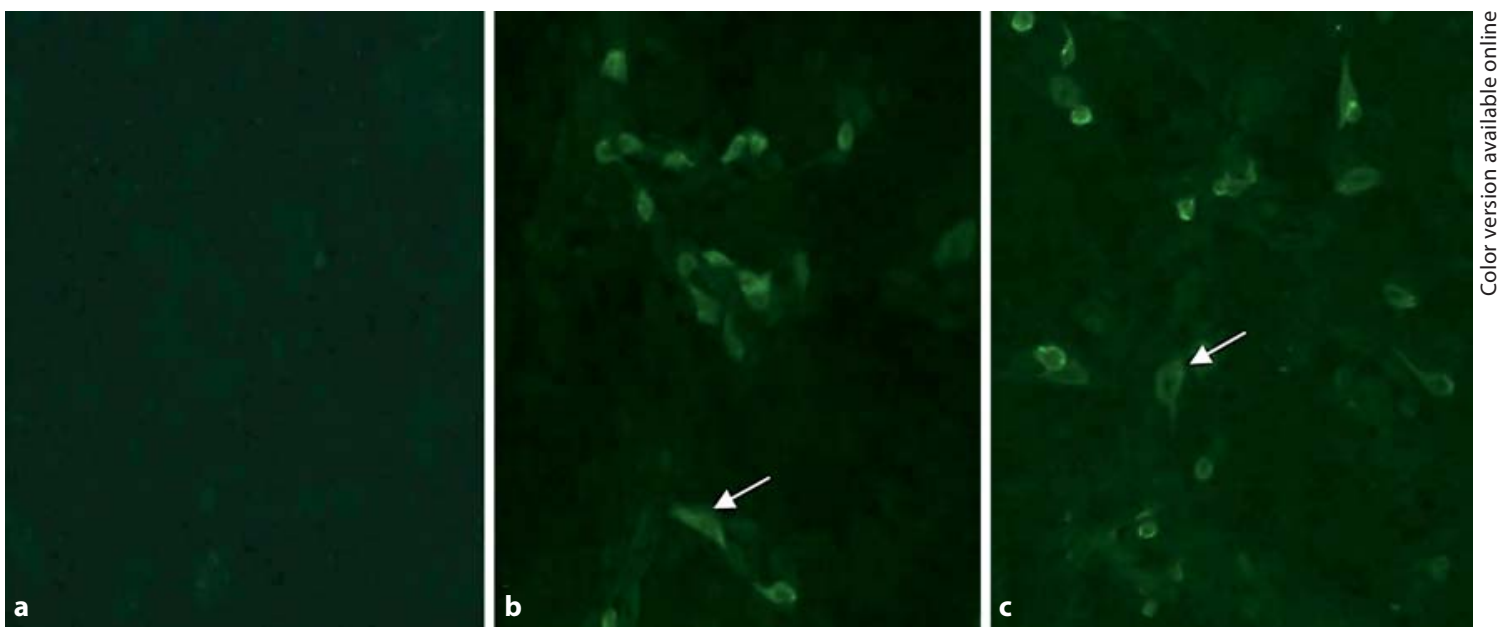

Fig. 6. Immunofluorescence analysis of influenza virus CG-infected MDCK cells. MDCK cells were infected with CG for $12 \mathrm{~h}$ and analyzed by immunofluorescence assay using negative murine serum (a), immune murine serum (b) and commercial influenza A M2 mAb (14C2; c), respectively.

High M2e Epitope Density Provided Partial Protection to Mice and Chickens against CG Virus Challenge,

Especially the MBP-3·M2e Fusion Protein

To evaluate the effect of high M2e epitope density on protective efficacy, lethal challenge assays in chickens and mice were carried out simultaneously with CG virus. As shown in figure 7, all fusion proteins used as antigen could delay the onset of disease in mice and chickens, compared with the rapid death that occurred in the negative control group in both models. In addition, the mice immunized with MBP-3.M2e fusion protein lost weight more slowly and recovered faster than mice in the other groups (fig. $7 \mathrm{c}$ ). Only MBP-3·M2e provided mice with a $16.7 \%$ survival rate, compared with $0 \%$ survival in the other groups. However, in the chicken model, MBP$3 \cdot \mathrm{M} 2 \mathrm{e}$ provided chickens with $37.5 \%$ survival, in comparison with the $12.5 \%$ survival in the groups given MBP$6 \cdot M 2 e, M B P-9 \cdot M 2 e$ and MBP-12・M2e. In contrast, the negative control group showed severe clinical signs and died 2-5 days after infection. In addition, the MBP-M2e group did not receive effective protection and all had died on the 9th day.

In terms of virus shedding, live viruses were isolated from the oropharyngeal and cloacal excreta of chickens, using the HA test in SPF embryonated chicken eggs. The results indicated that virus shedding was postponed in all groups immunized with the fusion proteins; among them, the MBP-3·M2e group displayed the least shedding, but shedding was not blocked in chickens of the negative control group (data not shown).

\section{Discussion}

As shown by the M2e constructs (table 1), two glycine residues are present in the 17th and 19th positions of the sequence, representing substitution of glycine for cysteine (C-G). This was aimed to overcome the drawback that these cysteines could lead to potential protein aggregation under oxidative conditions by the formation of interparticle disulfide bonds. At the same time, given that codon usage has been identified as the single most important factor in prokaryotic gene expression [20], codons were optimized in the construction to be beneficial for protein expression in both prokaryotic and eukaryotic systems, to ensure suitability for subsequent research in baculovirus-insect expression systems. Both treatments aim to enhance the stability of protein expression; in addition, the pMAL protein fusion and purification system was chosen to give higher levels of expression of soluble protein. Another construction of $n \cdot M 2 e$ plasmids with the pET30a vector (EMD Chemicals, Gibbstown, N.J., USA) was tested simultaneously, but was unable to express the fusion proteins (data not shown). Good expression was obtained with the pMAL system.

In order to get the best balance between protective efficacy and safety in the development of M2e-based vaccines, the use of an appropriate adjuvant is emphasized. The adjuvant is required to modulate the performance of M2e-based vaccines, in terms of increasing immunogenicity, and enhancing the immune response and vaccine potency $[21,22]$. Montanide ISA 70 M VG, water-in-oil 
emulsion appears to stimulate innate immunity and induce a delayed-type immune response with a longer duration of antibody response. The efficacy of this adjuvant was evaluated in our previous study of $\mathrm{rH} 5 \mathrm{~N} 3$ subtype AIV cell-cultured vaccine applied to SPF chickens and ducks [23]. Therefore, the same adjuvant was adopted in this study to improve the protective efficacy of the subunit vaccines.

Several studies have shown that M2e-specific antibodies can induce protection against influenza infection in vivo [24-26], but the mechanism of protection endowed by M2e-specific antibodies has not yet been confirmed. The present construct was chosen mainly on the premise that the protective efficacy of $n \cdot M 2 e$ fusion proteins is due to B cells and M2e-specific antibodies [27]. Measurement of the M2e antibody titers by ELISA only using the M2e-MAP peptide as antigen could not confirm that these specific antibodies can interact with native pathogen proteins. Cells infected with influenza virus, on which M2e proteins are expressed abundantly, may be the preferential target of antibodies in comparison with M2 proteins on virus particles $[5,28]$. Therefore, chickens and mice sera were detected with purified influenza virus particles, M2e-MAP peptide and the virus-infected MDCK cells as antigen, respectively. The results of ELISA with purified influenza virus particles and the native M2 proteins on MDCK cells guaranteed that the affirmative interaction of IgG with native M2 proteins and the antibody titers with the MDCK cell ELISA were higher than two others (fig. 6).

In the lethal challenge study, we used a fairly mild viral challenge in chickens and mice to evaluate the protective efficacy of M2e-based vaccination. Protection was assayed by the survival rate in chickens (fig. 7a), and the survival rate and weight loss in mice (fig. 7b, c). It may be noted that the challenge conditions used by many investigators to evaluate protective efficacy vary over a very wide range [24, 25]. In this study, the challenge dose was within the range used by other tests to assess the rate of mortality. It was clearly observed in this study that the protective efficacy in chickens and mice was incomplete, but many other researchers have obtained better protec-

Fig. 7. Chickens and mice immunized with different $n \cdot M 2 e$ fusion proteins were protected against CG challenge to different degrees. Monitoring of morbidity and lethality in SPF chickens (a) and $\mathrm{BALB} / \mathrm{c}$ mice by body weight loss (b) and survival rate (c).
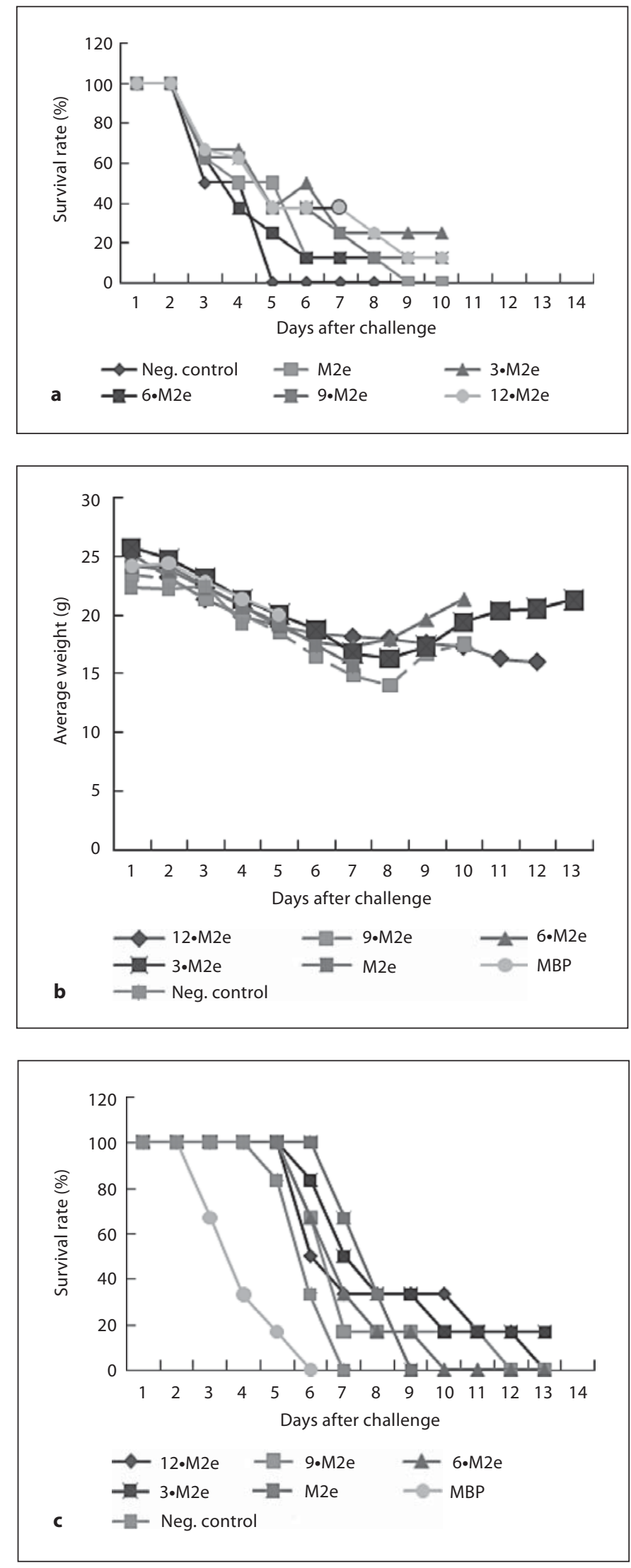

Intervirology 2011;54:290-299 
tion. There are many potential reasons for the diversity in the level of protection, such as the route of immunity, the challenge dose, the measurement of antibody titers and the strain of influenza virus used for challenge. Thus, it is not meaningful to make comparisons of the protective efficacy among experiments with different constructs. In addition, it was interesting in the present study that the $\mathrm{MBP}-3 \cdot \mathrm{M} 2 \mathrm{e}$ fusion protein rendered the best protection after challenge when compared with the results reported by Liu et al. [18], for which there are several possible explanations. First, they chose the human influenza virus $\mathrm{A} / \mathrm{PR} / 8 / 34$, while in the present study the influenza virus CG was used, in which the M2e sequence is different. Moreover, our previous study demonstrated that the influenza virus CG was highly pathogenic to both chickens and mice [29]; in our study, the survival of immunized chickens was prolonged. Third, the MBP tag, together with an unknown change in structure or conformation, may influence the presentation of the antigenic epitope, and may prevent sufficient interaction with immune cells. An additional reason could be that chickens instead of mice were challenged; the immune mechanism in the chicken model was different from that in the mouse model.

In conclusion, our study demonstrated a certain degree of protective efficacy of M2e-based subunit vaccines in chickens, which was different from that in the mouse model; in our study the MBP-3.M2e vaccine provided the best protection. It will be worthwhile to evaluate the immune response further in the chicken model with other epidemic influenza A isolates, and with other optimized vaccines that present M2e preferentially.

\section{Acknowledgments}

We thank Prof. Q. W. Meng for his help in the process of our animal experiment, and T. Zhang and other staff members for devoted animal care. This work was supported by grants from the 11th Five-year Support Programs for Science and Technology Development of China (2006BAD06A05) and the Natural Science Foundation of the Heilongjiang Province (ZJN-0702-02).

\section{References}

1 Chen H, Bu Z: Development and application of avian influenza vaccines in China. Curr Top Microbiol Immunol 2009;333:153-162.

2 Steinhauer DA, Skehel JJ: Genetics of influenza viruses. Annu Rev Genet 2002;36:305332.

-3 Subbarao K, Murphy BR, Fauci AS: Development of effective vaccines against pandemic influenza. Immunity 2006;24:5-9.

-4 De Filette M, Min Jou W, Birkett A, Lyons K, Schultz B, Tonkyro A, Resch S, Fiers W: Universal influenza A vaccine: optimization of M2-based constructs. Virology 2005;337: 149-161.

5 Fan J, Liang X, Horton MS, Perry HC, Citron MP, Heidecker GJ, Fu TM, Joyce J, Przysiecki CT, Keller PM, Garsky VM, Ionescu R, Rippeon Y, Shi L, Chastain MA, Condra JH, Davies ME, Liao J, Emini EA, Shiver JW: Preclinical study of influenza virus A M2 peptide conjugate vaccines in mice, ferrets, and rhesus monkeys. Vaccine 2004;22:2993-3003.

6 6 De Filette M, Martens W, Smet A, Schotsaert M, Birkett A, Londono-Arcila P, Fiers W, Saelens X: Universal influenza A M2e-HBc vaccine protects against disease even in the presence of pre-existing anti-HBc antibodies. Vaccine 2008;26:6503-6507.
7 Mozdzanowska K, Feng J, Eid M, Kragol G, Cudic M, Otvos L Jr, Gerhard W: Induction of influenza type A virus-specific resistance by immunization of mice with a synthetic multiple antigenic peptide vaccine that contains ectodomains of matrix protein 2 . Vaccine 2003;21:2616-2626.

-8 Eliasson DG, El Bakkouri K, Schon K, Ramne A, Festjens E, Lowenadler B, Fiers W, Saelens X, Lycke N: CTA1-M2e-DD: a novel mucosal adjuvant targeted influenza vaccine. Vaccine 2008;26:1243-1252.

-9 De Filette M, Ramne A, Birkett A, Lycke N, Lowenadler B, Jou WM, Saelens X, Fiers W: The universal influenza vaccine M2e-HBc administered intranasally in combination with the adjuvant CTA1-DD provides complete protection. Vaccine 2006;24:544-551.

10 De Filette M, Fiers W, Martens W, Birkett A, Ramne A, Lowenadler B, Lycke N, Jou WM, Saelens X: Improved design and intranasal delivery of an M2e-based human influenza A vaccine. Vaccine 2006;24:6597-6601.

11 Heinen PP, Rijsewijk FA, de Boer-Luijtze EA, Bianchi AT: Vaccination of pigs with a DNA construct expressing an influenza virus M2nucleoprotein fusion protein exacerbates disease after challenge with influenza A virus. J Gen Virol 2002;83:1851-1859.
12 Price GE, Soboleski MR, Lo CY, Misplon JA, Pappas C, Houser KV, Tumpey TM, Epstein SL: Vaccination focusing immunity on conserved antigens protects mice and ferrets against virulent $\mathrm{H} 1 \mathrm{~N} 1$ and $\mathrm{H} 5 \mathrm{~N} 1$ influenza A viruses. Vaccine 2009;27:6512-6521.

$\checkmark 13$ Ito T, Gorman OT, Kawaoka Y, Bean WJ, Webster RG: Evolutionary analysis of the influenza A virus $M$ gene with comparison of the M1 and M2 proteins. J Virol 1991;65: 5491-5498.

14 Lamb RA, Zebedee SL, Richardson CD: Influenza virus M2 protein is an integral membrane protein expressed on the infected-cell surface. Cell 1985;40:627-633.

15 Liu W, Zou P, Chen YH: Monoclonal antibodies recognizing EVETPIRN epitope of influenza A virus M2 protein could protect mice from lethal influenza A virus challenge. Immunol Lett 2004;93:131-136.

16 Fiers W, De Filette M, El Bakkouri K, Schepens B, Roose K, Schotsaert M, Birkett A, Saelens X: M2e-based universal influenza A vaccine. Vaccine 2009;27:6280-6283.

17 Liu W, Zou P, Ding J, Lu Y, Chen YH: Sequence comparison between the extracellular domain of M2 protein human and avian influenza A virus provides new information for bivalent influenza vaccine design. Microbes Infect 2005;7:171-177. 
18 Liu W, Peng Z, Liu Z, Lu Y, Ding J, Chen YH: High epitope density in a single recombinant protein molecule of the extracellular domain of influenza A virus M2 protein significantly enhances protective immunity. Vaccine 2004;23:366-371.

19 Liu ZQ, Chen YH: Design and construction of a recombinant epitope-peptide gene as a universal epitope-vaccine strategy. J Immunol Methods 2004;285:93-97.

-20 Gustafsson C, Govindarajan S, Minshull J: Codon bias and heterologous protein expression. Trends Biotechnol 2004;22:346-353.

-21 Leenaars PP, Hendriksen CF, Angulo AF, Koedam MA, Claassen E: Evaluation of several adjuvants as alternatives to the use of Freund's adjuvant in rabbits. Vet Immunol Immunopathol 1994;40:225-241.

-22 Leenaars PP, Hendriksen CF, Koedam MA, Claassen I, Claassen E: Comparison of adjuvants for immune potentiating properties and side effects in mice. Vet Immunol Immunopathol 1995;48:123-138.
23 Liu CG, Liu M, Wan CH, et al: Evaluation of adjuvant effect on $\mathrm{rH} 5 \mathrm{~N} 3$ subtype avian influenza virus cell-cultured vaccine in SPF chickens and ducks (in Chinese). Chin J Prev Vet Med 2009;31:217-221.

24 Rangel-Moreno J, Carragher DM, Misra RS, Kusser K, Hartson L, Moquin A, Lund FE, Randall TD: B cells promote resistance to heterosubtypic strains of influenza via multiple mechanisms. J Immunol 2008; 180:454463.

25 Nguyen HH, van Ginkel FW, Vu HL, McGhee JR, Mestecky J: Heterosubtypic immunity to influenza $A$ virus infection requires $B$ cells but not CD8+ cytotoxic T lymphocytes. J Infect Dis 2001;183:368-376.

26 Wang R, Song A, Levin J, Dennis D, Zhang NJ, Yoshida H, Koriazova L, Madura L, Shapiro L, Matsumoto A, Mikayama T, Kubo RT, Sarawar S, Cheroutre H, Kato S: Therapeutic potential of a fully human monoclonal antibody against influenza A virus M2 protein. Antiviral Res 2008;80:168-177.
27 Frace AM, Klimov AI, Rowe T, Black RA, Katz JM: Modified M2 proteins produce heterotypic immunity against influenza A virus. Vaccine 1999;17:2237-2244.

28 Jegerlehner A, Tissot A, Lechner F, Sebbel P, Erdmann I, Kundig T, Bachi T, Storni T, Jennings G, Pumpens P, Renner WA, Bachmann MF: A molecular assembly system that renders antigens of choice highly repetitive for induction of protective $\mathrm{B}$ cell responses. Vaccine 2002;20:3104-3112.

29 Ebrahimi SM, Tebianian M: Heterologous expression, purification and characterization of the influenza A virus M2e gene fused to Mycobacterium tuberculosis HSP70 (359$610)$ in prokaryotic system as a fusion protein. Mol Biol Rep 2009;37:2877-2883. 